Bond University

Research Repository

\title{
An Introduction to Temporal Optimisation using a Water Management Problem
}

Randall, Marcus; Montgomery, James; Lewis, Andrew

Published in:

Journal of Computational Science

DOI:

10.1016/j.jocs.2020.101108

Licence:

Other

Link to output in Bond University research repository.

Recommended citation(APA):

Randall, M., Montgomery, J., \& Lewis, A. (2020). An Introduction to Temporal Optimisation using a Water Management Problem. Journal of Computational Science, 42, [101108].

https://doi.org/10.1016/j.jocs.2020.101108

\section{General rights}

Copyright and moral rights for the publications made accessible in the public portal are retained by the authors and/or other copyright owners and it is a condition of accessing publications that users recognise and abide by the legal requirements associated with these rights.

For more information, or if you believe that this document breaches copyright, please contact the Bond University research repository coordinator. 


\title{
An Introduction to Temporal Optimisation using a Water Management Problem
}

\author{
M. Randall* \\ Bond Business School, Bond University, Queensland, Australia \\ J. Montgomery* \\ School of Technology, Environments and Design, University of Tasmania, Tasmania, Australia \\ A. Lewis* \\ Institute for Integrated and Intelligent Systems, Griffith University, Queensland, Australia
}

\begin{abstract}
Optimisation problems usually take the form of having a single or multiple objectives with a set of constraints. The model itself concerns a single problem for which the best possible solution is sought. Problems are usually static in the sense that they do not consider changes over time in a cumulative manner. Dynamic optimisation problems to incorporate changes. However, these are memoryless in that the problem description changes and a new problem is solved - but with little reference to any previous information. In this paper, a temporally augmented version of a water management problem which allows farmers to plan over long time horizons is introduced. A climate change projection model is used to predict both rainfall and temperature for the Murrumbidgee Irrigation Area in Australia for up to 50 years into the future. Three representative decades are extracted from the climate change model to create the temporal data sets. The results confirm the utility of the temporal approach and show, for the case study area, that crops that can feasibly and sustainably be grown will be a lot fewer than the present day in the challenging water-reduced conditions of the future.
\end{abstract}

\footnotetext{
${ }^{*}$ Corresponding author

Email addresses: mrandal1 abond. edu. au (M. Randall), james.montgomery@utas. edu. au (J. Montgomery), a. lewis@griffith. edu . au (A. Lewis)
} 
Keywords: Temporal modelling, Water resource management, Multi-objective Differential Evolution, Climate change

\section{Introduction}

All aspects of life, work and industry are driven by change. Change itself is dependent on the changes that have preceded it. This is far more than being dynamic, which implies a set of random changes without connection or a narrative between them. However, most optimisation problems are presented as static models, i.e., quantities are optimised given a single set of unchanging conditions [1]. While still computationally hard in the NP sense, they sometimes fail to adequately capture important aspects of the changing environment in which they operate. This paper therefore concentrates on the systematic incorporation of time into real world problems.

An excellent vehicle to demonstrate the importance and usefulness of the incorporation of temporal components in optimisation, are water resource management problems, as this commodity requires careful planning over time. In many parts of the world, water is a scarce commodity that has a wide variety of uses, from human and industrial consumption to agricultural and environmental purposes. As such, planning its correct and efficient use over long periods of time becomes necessary. Given the increasing importance of food security, the particular form of water resource management studied here is for agricultural (cropping) purposes [2, 3]. See Kurland and Zell [4] and Singh [5, 6, 7] for an overview and survey of these, as well as the wide variety and areas that water problems cover. Given that agricultural environments are subject to frequent change [8] and often involve long term considerations, modelling time in an appropriate manner is very important. Some examples of agricultural-oriented literature in which time is modelled with computational simulation are: Pluchinotta, Pagano, Giordano and Tsoukiàs [9], Tijskens, Ramon, and De Baerdemaeker [10] and Dent [11].

In this paper, a temporal framework for optimisation problems is developed. Using a temporally augmented crop planning problem, the performance of the problem and hence the framework is analysed using the well-known and tested optimisation approach, Differential Evolution (DE). This performance is tested on three decade-long data sets that have been produced using a known climate change model [12]. This will inform future temporal modelling efforts, not only in this domain, but others as well.

The remainder of this paper is organised as follows. Section 2 examines how the dimension of time has been used in optimisation problems and their modelling, 
with a focus on dynamic and water management problems. Section 3 presents a way to add a temporal component to one particular model. Section 4 describes the general principles of DE and how it is applied to the temporal oriented problem. For the problem under consideration, these concepts are tested empirically across decade long data sets in Section 5 with the multi-objective differential evolution. In Section 6 the implications of this work and future projects arising from it are discussed.

\section{The Dimension of Time in Optimisation Problems}

It has already been mentioned that time is an important factor in agricultural planning. The traditional construction of optimisation models, however, has concerned the issues of objectives (single, multiple or many), constraints and solution representations. The problems themselves in general require solvers (be they exact or heuristic based) to find the best possible solution, or set of trade-off solutions, in some computational time bound. The objectives may be linear or non-linear, but are not generally considered to have any temporal component, whereby an earlier part of the solution (at an earlier time period) could affect later decision making in the same solution vector. Such modelling is difficult as it requires one to project potential conditions into the future, which has a degree of uncertainty about it.

In this section, the closest analogue to temporal optimisation problems, namely the dynamic optimisation, is examined. Given the nature of water resource management issues, many problems in this domain consider time. This is done in an ad-hoc and non-systematic manner. As such, some of the more pertinent examples are considered here. From both of these groups, a working definition of temporal optimisation problems is developed.

\subsection{Dynamic Optimisation Problems}

The nearest analogues to optimisation problems that consider time are the dynamic optimisation problems [13]. According to Hendtlass, Moser and Randall [14] these are defined as having "an initial problem definition and a series of 'events' that change it over time. An event defines some change either to the data of the problem or its structural definition while the problem is being solved." (pp. 79-80). As such, these types of problems, while having a temporal component, are memoryless (i.e., they do not consider previous states in the determination of new states). New versions of the problem are progressively solved at various intervals. However, these are not built on in any cumulative way. This is an important 
point that will be elaborated on in the next section. The following briefly gives illustration to these points. Note that general surveys of dynamic optimisation and its applications may be found in Branke [13], Yang, Jiang and Nguyen [15] and Hendtlass, Moser and Randall [14].

The memoryless nature of dynamic optimisation is demonstrated by the following two typical works. Guntsch and Middendorf [16] use a population based ant colony optimisation (ACO) approach to solve dynamic versions of the travelling salesman problem (TSP) and quadratic assignment problem (QAP). For these two problems, the distance and flow data matrices change every $t$ iterations by allowing the insertion and deletion of $c$ cities/facilities. Both control variables are given a number of different values in independent runs of the ACO. When a change in problem definition occurs, ACO is required to find new solutions that satisfy and optimise the problem at hand. There is no memory of the previous problem or solutions.

Like the previous paper, Branke, Kaußler, Schmidt and Schmeck [17] develop techniques for adapting previous solutions to suit the new version of the problem. In their case, they use heterogeneous micro populations within a forking genetic algorithm to solve a benchmark dynamic problem, namely Moving Peaks. These micro populations, which they call "Self Organizing Scouts", track how potentially promising peaks move across the search landscape over time. Their approach significantly outperformed a standard genetic algorithm on the problem.

In contrast to the above, rather than the whole problem changing from one version to another, Mitra, Deb and Gupta [18] have a chemical quantity change in a continuous fashion. Particularly, they seek to minimise reaction time and the quantity of an undesirable compound, while the concentration of a monomer varies. This is part of a process to produce industrial quantities of nylon.

\subsection{Temporally Oriented Water Resource Management Problems}

Water, as a resource for agricultural purposes, often needs to be considered over extended time frames [19], with many papers referring to this issue. One work, Blanco, Fonseca and Flichman [20], puts forward some tentative notions concerning "inter-temporal" optimisation for natural resource problems. In this, they consider the effect of adjacent time periods in a cumulative manner. Moreover, their work considers individual decisions that then influence the decisions made later on. At each time step, the solver makes a decision between using stochastic dynamic programming or discrete stochastic programming.

The difference of this approach to the methods outlined in this paper is that the system and solver presented here make all of the temporal decisions simulta- 
neously, i.e., all crop areas and crop allocations are made across the entire planning horizon. It is anticipated that this will allow the solver greater latitude to find novel solutions that may have been excluded due to a staged approach. In other words, some solutions may have crop and water allocations in certain years that will temporarily make the objective values worse, only to potentially realise greater returns later on.

Other works considered here tend to fall into two categories of single and multiple time slices. For the former category, historical data are available. These usually concern water flows and availability over time. However, of all the data that are available, only a single time slice is analysed. Reed, Kollat and Devireddy [21] present a problem in which monitoring stations need to be optimally placed. They chose to use the data from eight years after a plume event. Naadimuthu, Raju and Lee [22] used a similar idea on an irrigation problem, except that they used a time differential between the sample time and the crop emergence time.

The category of multiple time slices builds on the above by considering a number of time slices in an historical data set, which are analysed separately. An example of this is by Rana, Khan and Rahimi [23] where different groundwater readings in both time and space were considered as part of the optimisation model. Again relationships are restricted to neighbouring slices and not in a holistic manner such as demonstrated herein.

\subsection{Temporal Optimisation Defined}

From the preceding discussions, certain characteristics of temporal optimisation problems should become evident. In many cases, a potential definition of temporal optimisation is informed by what has not been captured in prior related work. As such, an initial definition would be along the lines of "an optimisation problem in which all relevant temporal data is considered, as well as the interactions and cumulative effects of these data". In addition, one may wish to add that these problems would often take the form of predicting conditions into the future, given some measure of uncertainty. Indeed, the problem presented below is a case in point, as its data is based on climate change projections into the future.

\section{Temporal Optimisation using a Water Management Problem}

A variety of water resource management problems have been modelled and optimised in the literature. These range from groundwater management to crop planning $[24,25]$. It is the latter that is of primary concern here. Given the recent increases in global population and the subsequent shortages of adequate resources, 
any increase in the efficiency and the efficacy of crop production is of vital concern. One of the largest components of this is how water is used across various crops, and which crops should be planted to maximise some measure of utility (such as profit or social needs) while making sure that sufficient water is returned to the environment.

Prior to a description of the models on which the temporal framework is built, a discussion of the necessary modifications that need to be made is given. From these and the base problem (which only considers individual years), the development of the full temporal model is described.

\subsection{Agricultural Simplifying Assumptions}

While the model developed by Xevi and Khan [2] and then later refined and updated by Lewis and Randall [3] (as discussed next) contains many aspects of agricultural concern, there are other real agronomic issues that would need to be addressed for it to be used as part of a realistic planning tool for farmers or regional decision-makers. Bearing in mind that the stated aim of this work is to explore the dimension of time in optimisation problems, the following considerations will not be presently modelled:

- Crop Rotation - Broad-acre crops, such as cotton, rice, wheat, require periodic agronomic crop rotation as soil pathogens (like nematodes) and diseases build up, and therefore the land needs to be rested, or have a different crop sown. It must be noted as well that the model used in the previous papers and this one does not assign a crop to a specific geographical area.

- Growing Conditions of a Particular Crop - This includes sowing date, variety and irrigation strategy. Different options available as part of each of these will lead to different overall results, particularly depending on water availability. For the case study region, the Murrumbidgee Irrigation Area (MIA), these details are presented in Dunn and Gaydon [26] and Gaydon, Meinke and Rodriguez [27].

- Ground Conditions - The area under consideration, the MIA, is very large. This study and previous consider approximately 120,000 ha. Within this area, there are a variety of soil types and elevations that make them suitable for different crops. For example, broad-acre crops prefer large flat areas, whereas vines for wine grapes may ideally be placed on a slope of a hill.

At the present time, parallel work is proceeding in the incorporation of the above in an enlarged model. 


\subsection{Base Problem}

As mentioned above, the problem considered in this paper concerns the allocation of land to a subset of potential crops so as to maximise overall net revenue, while minimising the environmental flow deficit of the available water resources (from the surface and aquifers). The following reviews the development of the model.

Lewis and Randall [3] extended the work on Xevi and Khan [2] in the development of an optimisation model for this problem. Each crop had varying revenue potential, as well as watering requirements and variable costs. The authors extended the original work in two main ways: a) by extending and enhancing the underlying mathematical model and b) by applying a true multi-objective approach to produce a number of trade-off solutions. In terms of the former, the main enhancements were to: precisely model surface water versus ground water allocation; incorporate the variable costs into the net revenue objective; adding an objective for maintaining sufficient downstream flows for environmental purposes; limiting total groundwater pumping; and putting sensible planting area limits on perishable (and highly profitable) crops so that as much is being produced as the market for these crops can accept. Results of the model were realised through the use of the standard Non-Dominated Sorting Genetic Algorithm-II [28].

The Lewis and Randall [3] (pp. 181-182) model is reproduced in Equations 17. Using this, an examination of its components to determine the temporal aspects necessary to allow for planning across a number of years is undertaken.

$$
\begin{aligned}
& \text { Maximise } N R= \sum_{c=1}^{C} T C I(c) \times X(c) \\
&-C_{w} \times \sum_{m=1}^{M}\left(\left(\sum_{c=1}^{C} W R E Q(c, m) \times X(c)\right)-P(m)\right) \\
&-C_{p} \times \sum_{m=1}^{M} P(m) \\
&-\sum_{c=1}^{C} V \operatorname{cost}(c) \times X(c) \\
& \text { Minimise } E F D=\sum_{m=1}^{M}\left(T_{e n v_{-}} f(m)-E_{n} f(m)\right) \cdot\left[\operatorname{Env}_{-} f(m)<T e n v_{-} f(m)\right]
\end{aligned}
$$


s.t.

$$
\begin{gathered}
\sum_{m=1}^{M} P(m) \leq 50 \mathrm{GL} \\
\sum_{c=1}^{C} X(c) \leq \text { Tarea } \\
X(c) \leq Y(c) \quad 1 \leq c \leq C \\
\text { Allocation }(m)=\operatorname{Inflow}(m)-\text { Env_f }_{-}(m) \\
P(m)=\left(\sum_{c=1}^{C} \operatorname{WRE} Q(c, m) \times X(c)\right)-\operatorname{Allocation}(m)
\end{gathered}
$$

Where:

$N R$ is the net revenue,

$C$ is the number of crops,

$T C I(c)$ is the total crop income for $\operatorname{crop} c$,

$X(c)$ is a decision variable which is the area of crop $c$ (in hectares),

$C_{w}$ is total cost of water per unit volume $\$ / \mathrm{ML}$,

$M$ is the number of months, i.e., 12 ,

$W R E Q(c, m)$ is the water required for crop $c$ in month $m$ (in ML),

$P(m)$ is the groundwater pumped in month $m$,

$C_{p}$ is the cost of groundwater pumping and delivery (in $\$ / \mathrm{ML}$ ),

$V \operatorname{cost}(c)$ are all other variable costs associated with crop $c$,

$E F D$ is the deficit in environmental flow,

$T e n v_{-} f(m)$ is the target environmental flow for month $m$,

$E n v_{-} f(m)$ is a decision variable that is the environmental flow for month $m$, 
$T_{\text {Area }}$ is the total cropping area available,

$Y(c)$ is a the maximum allowable area for $\operatorname{crop} c$,

Allocation $(m)$ is the amount of surface water available for irrigation of crops in month $m$ and

Inflow $(m)$ is the total surface (river) water available in month $m$.

So far, this model is the basis of three other works. Lewis, Randall, Capon and Jackwitz [29] refined the model and data to more tightly and appropriately control water flow releases back to the environment. Montgomery, Fitzgerald, Randall and Lewis [30] also used this model to a test a Differential Evolution technique using various solution representation methods. Lewis, Randall, Elliot and Montgomery [31] coupled it with climate change prediction models in order to determine the types of crops that could still be grown in the next five decades, given changed water conditions.

\subsection{A Temporal Model for Water Management}

The model above assumes that there is only one year of planting. In reality however, some crops (i.e., the perennials) exist for a number of years. For example, fruit-bearing trees are grown over a number of years, reaching full maturity and yield after several seasons. Consequently, the problem should be solved over a longer planning horizon. To transform this existing model into one consistent with the definition of temporal optimisation given in Section 2.3, the objectives in the first instance need to determine the commercial value of the crops across all years, as well as the impact on water resources and hence the environment. Equation 8 remodels the net revenue calculations so that it ranges across different plantings of different crops in a defined planning horizon. 


$$
\begin{aligned}
& \operatorname{Max} . N R=\sum_{c=1}^{C}\left(\sum_{h=1}^{\max (x(c))} \sum_{y=1}^{Y}\left\{\begin{array}{ll}
i=0 & \text { if } h>x(c, y), \\
i=i+1 & \text { otherwise. }
\end{array} \quad T C I(c, y) \times S(i)\right)\right. \\
& -\sum_{y=1}^{Y}\left(C_{w}(y) \times \sum_{m=1}^{M}\left(\sum_{c=1}^{C}(W R E Q(c, m, y) \times X(c, y))-P(m, y)\right)\right) \\
& -\sum_{y=1}^{Y}\left(C_{p}(y) \times \sum_{m=1}^{M} P(m, y)\right) \\
& -\sum_{y=1}^{Y} \sum_{c=1}^{C} V \operatorname{cost}(c, y) \times X(c, y) \\
& -\sum_{y=1}^{Y} \sum_{c=1}^{C} \begin{cases}(X(c, y)-X(c, y-1)) \times E(c, y) & \text { if } X(c, y)>X(c, y-1) \\
0 & \text { otherwise }\end{cases} \\
& -\sum_{y=1}^{Y} \sum_{c=1}^{C} \begin{cases}(X(c, y-1)-X(c, y)) \times R(c, y) & \text { if } X(c, y-1)>X(c, y) \\
0 & \text { otherwise }\end{cases}
\end{aligned}
$$

Note that $Y$ is total number of years. As can be seen from Equation 8, quite a few changes and additions are necessary to account for time, and particularly potential crop changes over the years. These new elements mainly concern the planting and removal of crops from year to year, which not only attracts a direct cost, but can also result in differing yields as crops mature. The first line of Equation 8 calculates the crop income dependent on the levels of planting over the planned time horizon. This is due to crops maturing at different rates coupled with the different land areas that can be assigned to them over time. To this end, the term $S(c, k)$ is the proportion of the return for crop $c$ in the $k^{\text {th }}$ year after it has been planted $(0 \leq S(c, y) \leq 1)$. This means that for a crop like vines, an income may only be gained after a certain number of years. For example, if $S($ Vines $)=\{0,0,0,0,1\}$ a vineyard's produce would be ready for harvest (and hence potentially for sale) in the fifth year after planting. An annual plant such as lucerne [32], would have consistent values of 1 of course. Other crops like citrus may be at say half capacity after a number of years have elapsed, hence a value of 0.5 would be used. Another way of thinking about this is that $S$ is essentially the discount factor and applies to the correct land areas as they change over time. For example, if for a particular crop, there are 80 hectares in the first year and 100 
in the second year, $S=(0.1,0.5)$ and $T C I=(100,120)$, the calculations would become:

$$
\begin{gathered}
\text { Year } 1 \\
80 \times 0.1 \times 100=800 \\
\text { Year } 2 \\
(100-80) \times 0.1 \times 120=240 \\
+80 \times 0.5 \times 120=4800 \\
\text { Total }=\$ 5840
\end{gathered}
$$

For the purposes of this first instantiation of the temporal model, and as a simplifying assumption, in the case that a crop size decreases from one year to the next, the removed part of the crop is assumed to be the newest planted.

Lines 2 to 4 of Equation 8 simply add in the extra dimension of time to these parts. However, monetary values such as $C_{w}(y)$ and $C_{p}(w)$ can now change with factors like inflation. Lines 5 and 6 calculate the establishment and removal costs respectively. These costs will vary according to the crop and again apply to the perennials. For example, removing well established grape vines is a very costly exercise, whereas planting spring vegetables is relatively cheap. In these lines, costs are relative to the amount being established (or removed) from a particular year. This helps to explain the nature of the $X$ decision variable which now allows a crop's land area to vary dynamically over a set number of years. Note that $X(c, 0)=0, \forall C, 1 \leq c \leq C$ as planting starts in year 1 and it is assumed, for the sake of simplicity, that only bare land exists beforehand.

Min. $E F D=\sum_{y=1}^{Y} \sum_{m=1}^{M}\left(\operatorname{Tenv}_{-} f(m, y)-E n v_{-} f(m, y)\right) \cdot\left[E n v_{-} f(m, y)<T e n v_{-} f(m, y)\right]$

The constraints vary less dramatically than the objectives and simply require the extra dimension of time to be added to each. However, by doing this, it allows the model to cater for variations across the years. Consider, for example, Constraint 11, which allows planners to vary the available groundwater pumping from year to year, based on future projections.

$$
\begin{gathered}
X(c, y) \leq L(c, y) \quad \forall c, 1 \leq c \leq C \quad \forall y, 1 \leq y \leq Y \\
\sum_{m=1}^{M} P(m, y) \leq P t(y) \quad \forall y, 1 \leq y \leq Y
\end{gathered}
$$




$$
\sum_{c=1}^{C} X(c, y) \leq \text { Tarea } \quad \forall y, 1 \leq y \leq Y
$$

$\operatorname{Allocation}(m, y)=\operatorname{Inflow}(m, y)-E_{n} v_{-} f(m, y) \quad \forall m, 1 \leq m \leq M \quad \forall y, 1 \leq y \leq Y$

$$
\begin{array}{r}
P(m, y)=\max \left(\left(\sum_{c=1}^{C} W R E Q(c, m, y) \times X(c, y)\right)-\operatorname{Allocation}(m, y), 0\right) \\
\forall m, 1 \leq m \leq M \quad \forall y, 1 \leq y \leq Y
\end{array}
$$

Where:

$T C I(c, y)$ is the total crop income for crop $c$ in year $y$,

$X(c, y)$ is a decision variable which is the area of crop $c$ in year $y$ (in hectares),

$\operatorname{WREQ}(c, m, y)$ is the water required for crop $c$ in month $m$ of year $y$ (in $\mathrm{ML})$,

$C_{w}(y)$ is total cost of water per unit volume $\$ / \mathrm{ML}$ in year $y$,

$C_{p}(y)$ is the cost of groundwater pumping and delivery $\$ / \mathrm{ML}$ in year $y$,

$V \operatorname{cost}(c, y)$ are all other costs associated with crop $c$ in year $y$,

$E n v_{-} f(m, y)$ is a decision variable that is the environmental flow for month $m$ in year $y$,

$S(c, y)$ is the proportion of the return for crop $c$ in the $y^{\text {th }}$ year after it has been planted $0 \leq S(c, y) \leq 1$,

$E(c, y)$ is the establishment cost of crop $c$ per hectare in year $y$,

$R(c, y)$ is the removal cost of crop $c$ per hectare in year $y$,

$T e n v_{-} f(m, y)$ is the target environmental flow for month $m$ in year $y$,

$P(m, y)$ is the groundwater pumped in month $m$ in year $y$, 
$\operatorname{Pt}(y)$ is the pumping target (in gigalitres) for year $y$. In the previous study this was set as 50GL.

$\operatorname{Inflow}(m, y)$ is the total surface (river) water available in month $m$ in year $y$,

Allocation $(m, y)$ is the amount of surface water available for irrigation of crops in month $m$ of year $y$ and

$L(c, y)$ is the maximum area that crop $c$ is limited to in year $y$.

\section{Using Differential Evolution as the Optimisation Engine}

DE has been a highly successful evolutionary algorithm ${ }^{1}$ across a range of problems and has been applied to the single year version of this problem previously $[30,31]$. In terms of the single year problem [30], it showed that it was able to outperform platforms like NSGA-II (see, e.g., [28]). It uses a population of solutions to optimise over a number of generations. Like genetic algorithms, crossover and mutation are responsible for the manipulations in the algorithm. However, they are used in quite a different way to other evolutionary algorithms. Mutation is typically not a simple operation in which a single solution component is changed, but one based on often complex recombination between a number of solution vectors. DE's naming convention $\mathrm{DE} / a / b / c$ denotes a $\mathrm{DE}$ algorithm for which $a$ and $b$ describe how mutation is carried out and $c$ is for the crossover type. One of the most common variants, that used herein, is DE/rand/1/bin (see, e.g., Price, Storn and Lampinen [33] for details).

The multi-objective DE used here is based on that used by Montgomery, Randall and Lewis [34] for RFID antenna design that uses the mutation mechanism of DE coupled with the non-dominated sorting routine from NSGA-II. The search begins by creating a population of solution vectors. At every generation, each solution vector is recombined (mutated) and crossover is performed using DE solution mutation mechanisms. In DE/rand/1/bin, three other members are chosen to be recombined with the current member. A solution component of a child vector is probabilistically generated from all these vectors according to Equation 15. A

\footnotetext{
${ }^{1}$ Note that evolutionary algorithms do not guarantee that the optimal solution will be obtained, or for multi-objective optimisation, that the true Pareto front will be reached. They will, however, find good solutions or attainment surfaces for non-convex problems in reasonable amounts of computational time, which is why they are used in this work.
} 
solution component is each separate value in the solution vector. For the problem used in this paper, the assignment of an area of land (in hectares) to a particular crop in a particular year as well as a water allocation per month per year, represent single components.

$$
\text { child }_{j}=x_{r_{3} j}+F\left(x_{r_{1} j}-x_{r_{2} j}\right)
$$

Where:

child $_{j}$ is the $j^{\text {th }}$ component of the child vector,

$x_{a b}$ is the $b^{\text {th }}$ component of population member $a$,

$r_{1 \ldots 3}$ are three solution vector indexes defining the other members with which the solution is to be recombined, and

$F$ is the scaling factor.

$F$ governs the amount of diversity that is introduced. A high value (around 1) corresponds to a large diversity and visa-versa. Equation 15 is invoked from a probabilistic rule with its associated parameter value $C R$ (the crossover rate). If it is not used, the original solution component is directly added to the child. Occasionally, the computed value may fall outside the problem defined boundaries. The boundary checking rule is given in Equation 16.

$$
\text { child }_{j}= \begin{cases}\frac{x_{i j}+l b_{i}}{2} & \text { if } \text { child }_{j}<l b_{i} \\ \frac{x_{i j}+u b_{i}}{2} & \text { if child } \text { ch }_{j}>u b_{i} \\ \text { child }_{j} & \text { otherwise }\end{cases}
$$

Where:

$i$ is the index of the parent solution vector,

$l b_{i}$ is component $i$ 's lower bound and

$u b_{i}$ is component $i$ 's upper bound.

In the case of multi-objective optimisation, such as for this problem, a child is kept if it dominates any solution in the archive. ${ }^{2}$ The final membership of the archive is used to produce the attainment surfaces that will be given in Section 5.

\footnotetext{
${ }^{2}$ The archive is a set of non-dominated solutions that are updated across the generations of the search. The size of this is set as the population size.
} 


\subsection{Producing Initial Solutions}

For all iterative based optimisation algorithms, such as DE, an initial solution (or multiple if it is a population technique) needs to be generated. Of course, for any particular problem, there are multiple ways in which this can be done. For this work, land areas for the crops under consideration are generated at random. Approximately half are left at zero (so not selected) to help ensure that land is not over allocated. Thus, the areas, as close as possible, sum up to Tarea.

\section{Computational Experiments}

To determine how the previously described material and ideas work in practice, appropriate data sets are constructed and then tested using the described optimisation framework. From the results gained the effects of time are drawn out and compared to solutions gained in the previously published single year studies. One of the key points of interest will be to see the difference in crop mixes between them.

\subsection{Constructing Decade Long Data Files}

Given that this paper proposes a new form of problem class, considerable effort is required to develop appropriate test problems that will adequately explore the model. In order to extend the existing data files, as used in Lewis and Randall [3], so that they have a temporal component, five major issues need to be addressed:

1. Water Requirements - These depend on both rainfall and temperature values. Modelling these, even in the short to medium term, is difficult given the various methods of climate change prediction. Nonetheless, Evans, Ji, Lee, Smith, Argüeso and Fita [12], develop 12 different climate models for the regions of New South Wales and the Australian Capital Territory. This is referred to as NARCliM. The 12 models are themselves ensembles of four Global Climate Models (GCMs) and three Regional Climate Models (RCMs). The GCMs and RCMs are chosen for overall effectiveness, and GCMS are downscaled by the RCMs. The models are then used to generate predicted rainfall and temperature over an area of interest (for this paper this is restricted to the MIA). The time boundaries that it is able to provide, that best serve this paper are between the years 1990 and 2070. The 12 models therefore give 12 scenarios over which to potentially run the temporal model. As in the work of Lewis, Randall, Elliot and Montgomery [31], the 
model delivering results closest to the mean of the ensemble of the NARCLiM models, CCMA3.1-R3, will be used to generate the different WREQ values over time. An additional consideration is the cost of pumping water from underground aquifers. This price varies according to whether a year is considered to be wet, average or dry, as detailed in the note on Year Classification in item 5 below.

2. Establishment and Removal costs - Depending on the biological nature and complexity of the crop, these costs will vary considerably. For example, vegetables will typically be planted by scattering or the use of planting machines, whereas grape vines require expensive root stocks. For annual crops, such as cotton, canola and vegetables, the establishment costs are incorporated into the variable costs ( $V$ cost in the temporal model) so are effectively $\$ 0$. Similarly, the removal costs correspond to harvesting and also are set at $\$ 0$. Approximate costings for the three perennial crops were sourced from Verdegaal, Klonsky, and de Moura [35], Hansen [36], Morgan and Olmstead [37], IFTA [38], TreeFruit [39], Van den Ende [40], DPIPWE [41] and O'Connell et al [42].

3. Crop Maturity Rates - This is somewhat related to the previous point. For the three perennial crops, it will take a number of years before they are fully mature, and will only produce a proportional amount of fruit, usually after an initial period. This corresponds to the $S$ variable in the temporal model. For grape vines, according to Verdegaal, Klonksky and de Moura [35], most wine grapes will start to bear fruit from three years with a yield of about 3-4 t/ha. This will increase to approximately $8 \mathrm{t} / \mathrm{ha}$ at around seven years and up to $12 \mathrm{t} / \mathrm{ha}$ at age 30 . Using these points, a logarithmic regression equation can be formed as $y=3.3954 \ln (x)+0.7046$, where $y$ is the yield per hectare and $x$ is the year after planting. For example, after five years, the vines will reach approximately $70 \%$ of their fruit bearing capacity. For most stone fruit, it takes at least three years to realise a full harvest [43].

4. Inflation - Any economic related system that has a temporal component must account for inflation. For ease of comparison in Section 5.3, all figures are expressed in 2020 Australian dollars. In the first instance, the figures used by Xevi and Khan [2] and Lewis and Randall [29] must be brought forward to this year. These were quoted in 2005 Australian dollars. This was done using Consumer Price Index (CPI) figures from the Reserve Bank of Australia [44]. CPI is set to the average of the years since 2005 which equates to $2.5 \%$. All monetary figures used by the model are indexed by this amount on an annual basis until 2020. Establishment and removal costs 
will be calculated relative to the publication dates where the values were sourced.

5. Year Classification - In order to determine the $C_{p}$ values, each year needs to be classified as either wet, average or dry. This is dependent on the predicted rainfall. The rationale is that pumped water will be more expensive in a dry year than it would be in a wet or average year, and vice versa. The amount of rainfall in the target area of the MIA also depends on the stream inflow coming from the nearby Snowy Mountains region. As such, the predicted rainfall across the two regions for all months was summed for each year. These were then summed and ranked. The lowest decile corresponded to the driest years, while the top decile were seen as wet years. The rest were classified as average. This approach is consistent with the method used by Xevi and Khan [2].

The three decade-long data files, as well as a template file, are available on request from the first author.

\subsection{DE Parameters}

In line with the work conducted by Montgomery et al. [30] and Lewis et al. [31], the following values have been given to the DE specific values: population size $=100, F=0.5$ and $C r=0.8$, with iterations $=30,000$ (based on the common heuristic of allowing $10 \mathrm{k} \cdot D$ function evaluations, where $D=280$ is the number of decision variables). The algorithm was run for 21 randomised trials on each problem instance.

\subsection{Computational Results and Analysis}

Figure 1 summarises the search progress for selected trials for the three decadelong problem instances, presenting the attainments surfaces from the initial population and at 10k, 20k and 30k iterations (as noted in the figure). The trials were selected as having a final attainments surface (i.e., iteration 30k) with median generational distance (GD) [45] (measured against an artificial Pareto front created by taking the non-dominated solutions across all 21 trials for each instance). ${ }^{3} \mathrm{Al}$ though not easily discernible due to the scale of the charts, the best solutions in the initial population include some with $N R<\$ 0$, indicating that while the solutions are feasible they are far from optimal. The charts show that the algorithm makes

\footnotetext{
${ }^{3}$ The distance calculation involves rescaling objective values to lie in the unit interval so that changes in each objective are the same magnitude.
} 


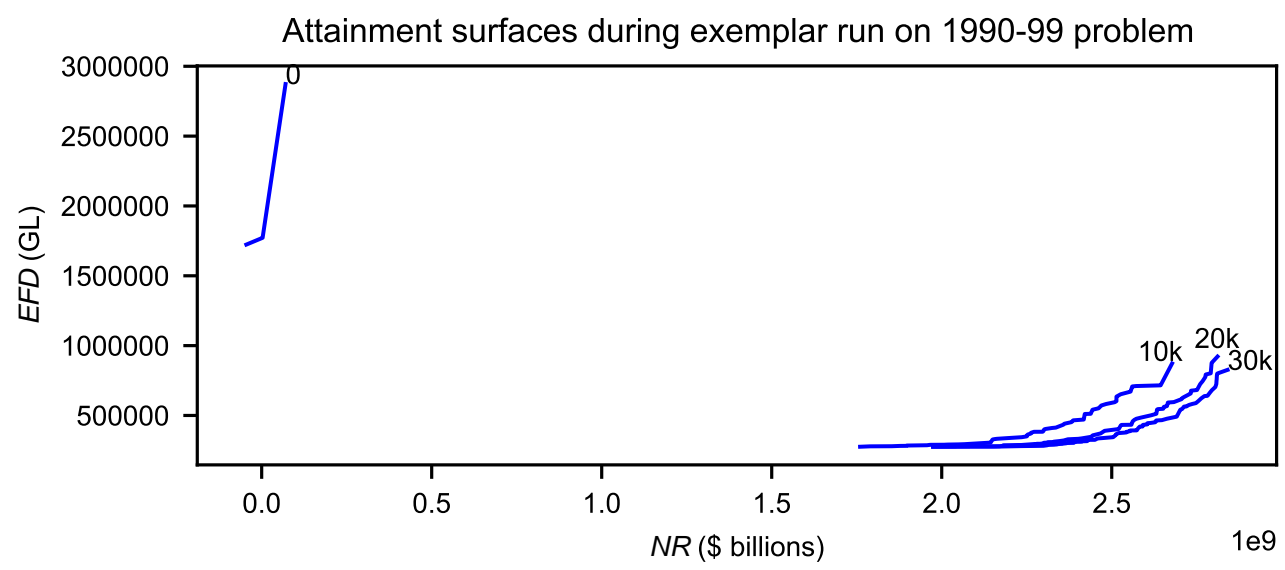

Attainment surfaces during exemplar run on $2020-29$ problem

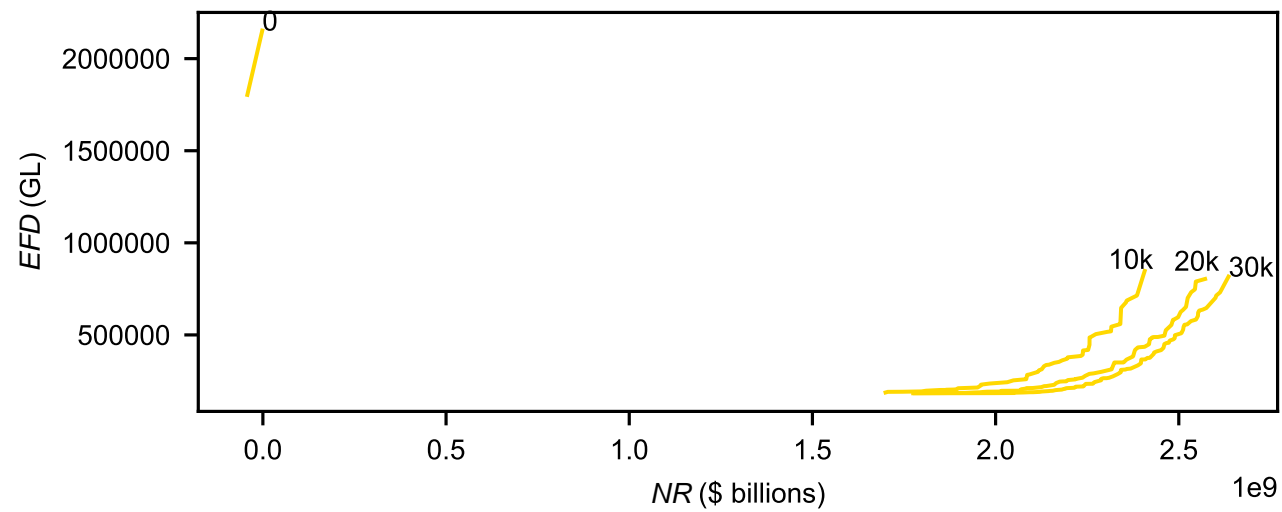

Attainment surfaces during exemplar run on 2060-69 problem

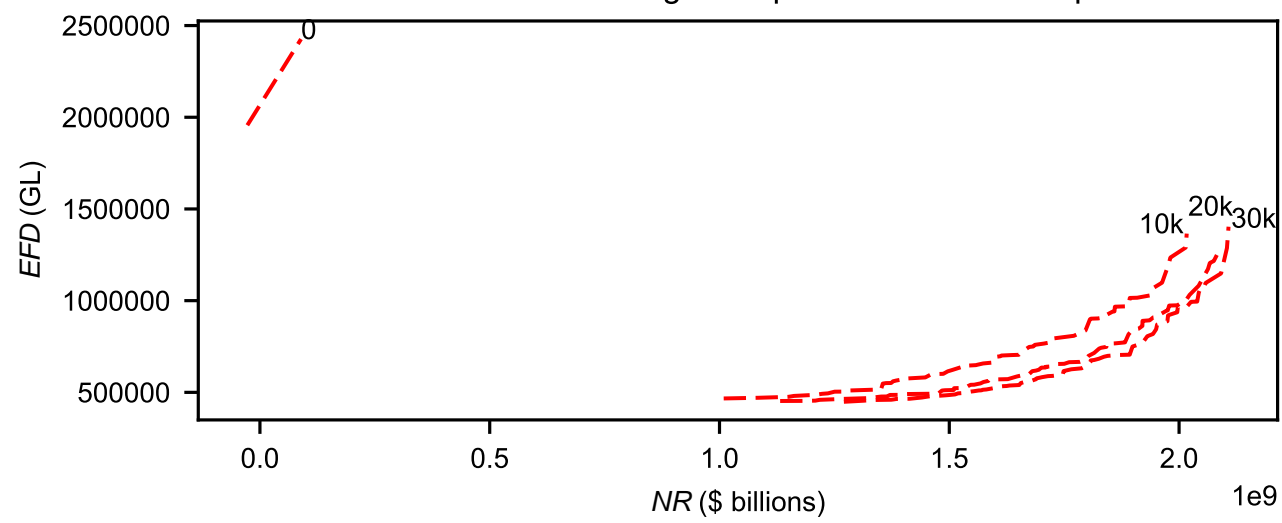

Figure 1: Attainment surfaces from representative trials by iteration for each problem. Note that the surfaces for iteration 0 , the initial population, contain only 3, 2 and 2 solutions, respectively. 


\begin{tabular}{rccc}
\hline & \multicolumn{3}{c}{ Decade } \\
\cline { 2 - 4 } Iteration & $1990-99$ & $2020-29$ & $2060-69$ \\
\hline 0 & {$[0.397,0.465]$} & {$[0.390,0.454]$} & {$[0.314,0.357]$} \\
10,000 & {$[0.014,0.019]$} & {$[0.016,0.020]$} & {$[0.008,0.011]$} \\
20,000 & {$[0.007,0.010]$} & {$[0.008,0.011]$} & {$[0.004,0.007]$} \\
30,000 & {$[0.004,0.007]$} & {$[0.005,0.008]$} & {$[0.003,0.005]$} \\
\hline
\end{tabular}

Table 1: 95\% confidence intervals on generational distance measure by instance and iteration

substantial progress during the first third of its search, with continuing, slower progress for the remainder of its allowed runtime. Table 1 presents the $95 \%$ confidence intervals on the calculated GD by problem and iteration sample point, and confirms that the typical performance of the search approaches the combined best performance (the mean GD values approach 0 ). Taken together this suggests that the search is converging to an approximation of the true Pareto front (which longer runs or the application of local search could then find).

Figure 2 presents the final attainment surfaces produced for the three decadelong problem instances for the selected trials, overlaid to allow for comparison between decades. For all decades, the attainment surfaces include at least one solution with the minimum achievable $E F D$ given actual or predicted inflows per month and the fixed target of 100,000 GL environmental flow in each month. Evidently, the forecast conditions for the 2060-69 decade mean target environmental flows will rarely if ever be met, even if planting very little (the lowest $E F D$ solution in the selected solution set leaves $48 \%$ of the area unallocated). Further, these results indicate that 2060-69 is likely to be significantly less profitable than what is achievable now and in the recent past.

Table 2 presents the crop allocations across decades for the minimum and maximum $N R$ solutions for each decade for the selected solution sets (the crop allocations are of greater interest than details of the environmental flows across each month). Also included are details for: solutions from the selected 1990 and 2020 attainment surfaces nearest where they cross in objective space (labelled B and $\mathrm{F}$ in Figure 2, respectively); and the solution from the 1990 solution set with equivalent $N R$ to the maximum $N R$ in the selected 2020 solution set, but with clearly superior $E F D$ performance (labelled C).

The summary allocation data presented in Table 2 shows that, regardless of decade or position within the attainment surface, certain crops are favoured by the 


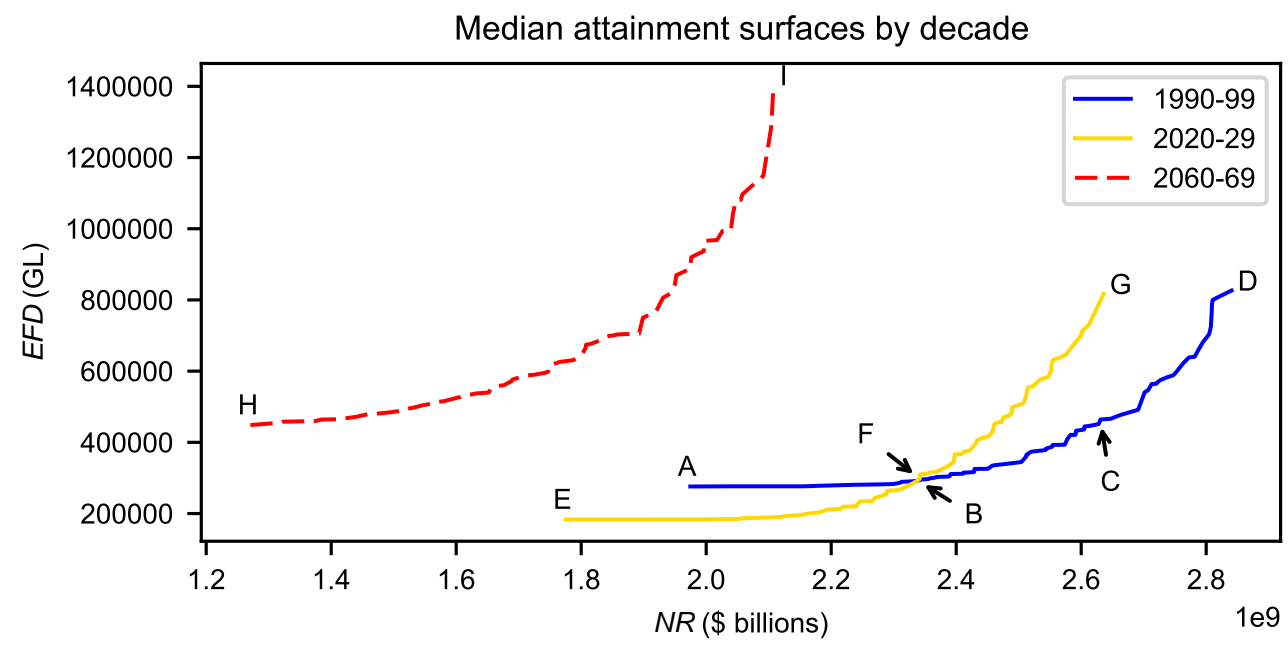

Figure 2: Representative attainment surfaces produced for each decade, selected as being the closest to the median Generational Distance across trials.

Table 2: Selected crop allocations from median attainment surfaces, rounded to the nearest 1000 ha. Values represent the total area in kha allocated across the decade-long planning horizon. IDs refer to labelled points in Figure 2.

\begin{tabular}{|c|c|c|c|c|c|c|c|c|c|c|c|c|c|c|c|c|c|c|c|}
\hline \multirow[b]{2}{*}{ ID } & \multirow[b]{2}{*}{$N R$} & \multirow[b]{2}{*}{$E F D$} & \multicolumn{16}{|c|}{ Total area allocated across decade (kha) } & \multirow[b]{2}{*}{ unalloc } \\
\hline & & & $\mathrm{R}$ & W & $\mathrm{B}$ & M & $\mathrm{Ca}$ & $\mathrm{O}$ & $S$ & WP & SP & $\mathrm{L}$ & $\mathrm{V}$ & WV & SV & $\mathrm{Ci}$ & $\mathrm{SF}$ & Co & \\
\hline \multicolumn{20}{|c|}{ Decade: $1990-99$} \\
\hline A & $\$ 1.97 \mathrm{~B}$ & $276.0 \mathrm{TL}$ & 57 & 34 & 19 & 3 & 355 & 119 & 8 & 19 & 9 & 17 & 7 & 22 & 6 & 8 & 5 & 178 & $28 \%$ \\
\hline B & $\$ 2.34 \mathrm{~B}$ & $292.5 \mathrm{TL}$ & 51 & 34 & 21 & 4 & 372 & 129 & 4 & 8 & 9 & 10 & 4 & 21 & 8 & 8 & 3 & 268 & $20 \%$ \\
\hline $\mathrm{C}$ & $\$ 2.63 \mathrm{~B}$ & $464.1 \mathrm{TL}$ & 52 & 41 & 17 & 5 & 356 & 122 & 4 & 13 & 8 & 5 & 3 & 22 & 9 & 8 & 3 & 371 & $13 \%$ \\
\hline $\mathrm{D}$ & $\$ 2.84 \mathrm{~B}$ & $826.4 \mathrm{TL}$ & 55 & 25 & 17 & 4 & 300 & 112 & 6 & 11 & 9 & 6 & 3 & 22 & 9 & 9 & 3 & 481 & $11 \%$ \\
\hline \multicolumn{20}{|c|}{ Decade: $2020-29$} \\
\hline $\mathrm{E}$ & $\$ 1.78 \mathrm{~B}$ & $183.2 \mathrm{TL}$ & 13 & 77 & 40 & 11 & 312 & 265 & 8 & 28 & 18 & 14 & 4 & 21 & 8 & 8 & 5 & 85 & $24 \%$ \\
\hline $\mathrm{F}$ & $\$ 2.34 \mathrm{~B}$ & $297.4 \mathrm{TL}$ & 22 & 36 & 25 & 5 & 381 & 306 & 4 & 9 & 10 & 7 & 2 & 22 & 10 & 8 & 3 & 202 & $12 \%$ \\
\hline G & $\$ 2.64 \mathrm{~B}$ & $816.7 \mathrm{TL}$ & 30 & 12 & 17 & 4 & 340 & 257 & 4 & 4 & 8 & 7 & 4 & 22 & 10 & 7 & 2 & 352 & $10 \%$ \\
\hline \multicolumn{20}{|c|}{ Decade: $2060-69$} \\
\hline $\mathrm{H}$ & $\$ 1.27 \mathrm{~B}$ & $448.6 \mathrm{TL}$ & 9 & 101 & 33 & 3 & 278 & 123 & 3 & 5 & 8 & 8 & 3 & 22 & 7 & 8 & 2 & 8 & $48 \%$ \\
\hline I & $\$ 2.11 \mathrm{~B}$ & 1.4 PL & 23 & 119 & 34 & 3 & 449 & 169 & 7 & 14 & 7 & 8 & 4 & 22 & 8 & 9 & 2 & 134 & $16 \%$ \\
\hline
\end{tabular}

Crops are (R)ice, (W)heat, (B)arley, (M)aize, (Ca)nola, (O)ats, (S)oybean, winter pasture (WP), summer pasture (SP), (L)ucerne, $(\mathrm{V})$ ines, winter vegetables $(\mathrm{WV})$, summer vegetables $(\mathrm{SV}),(\mathrm{Ci})$ trus, stone fruit $(\mathrm{SF})$ and $(\mathrm{Co})$ tton 
solver: cotton, canola, oats in the highest quantities, then barley and wheat, with rice occasionally being included. (The low NR solution from 2060-69 set is an exception, with very little cotton.) As in prior work [30] the key environmental trade-off is how much area is allocated to cotton, given its higher water requirement than the other high-value crop canola. Looking across the three planning decades, as water availability diminishes and average temperatures increase, the solver must reduce both the total area planted and the amount allocated to cotton, although canola, oats and wheat are still planted in relatively large quantities.

The crops with upper bounds applied (vines, winter and summer vegetables, citrus and stone fruit) are planted in highly similar proportions regardless of the planning decade. Vines are planted in very small quantities (typically only $3 \%$ of its upper bound), stone fruit typically one third its upper bound, and citrus and summer vegetables approximately $55-60 \%$ their limit. Winter vegetables are consistently planted near their upper limit, which is likely due to the anticipated inflow of water during winter months.

As DE is a stochastic solver there is variability in the final solution sets produced. For example, in the best of the produced attainment surfaces (details not included for simplicity of presentation), the solver produces less diverse crop allocations that strongly favour canola, but with consistent allocations of cotton in the 1990-99 and 2020-29 problems. The best trial for the 2060-69 decade follows this pattern but also includes a substantial amount of barley. Allocations of the bounded crops are almost identical to those in the selected exemplar results.

The 10-year planning horizon in the instances studied here means that crop allocations for vines, citrus and stone fruit in one year influence the expected return in subsequent years (these crops also have high planting and removal costs, particularly citrus trees). Although initial solutions allocate these crops with 50\% probability in any year, with no consideration of the year-on-year impact of those decision, the solver should take this into account. Figure 3 plots the areas allocated to these crops for selected solutions from the 'median' trial (left column) and best performing trial (right column). The plots suggest that the solver is able to arrive at relatively stable allocations for these crops in some cases, particularly citrus (which has the highest removal cost of all), indicating it is adapting to the temporal nature of the problem. However, there is still some degree of variability that could be further refined.

Those crops planted in the largest quantities (cotton, canola and oats), which have low planting and removal costs and which are at $100 \%$ yield within one season, tend to vary more between years, with average changes in allocated area $20-30 \%$ of the available space. In contrast, citrus and stone fruit tend to change 


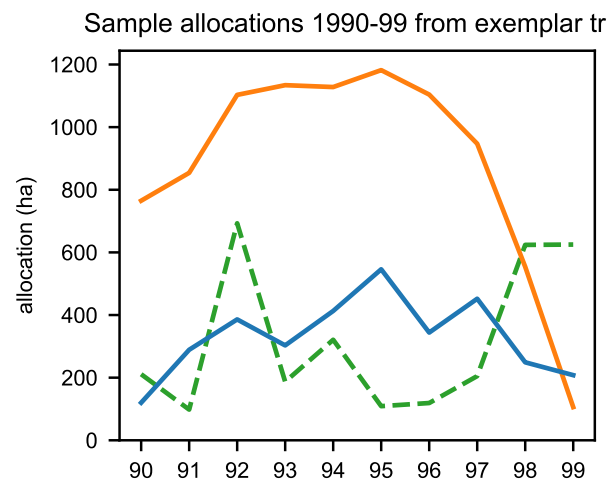

Sample allocations 1990-99 from best trial

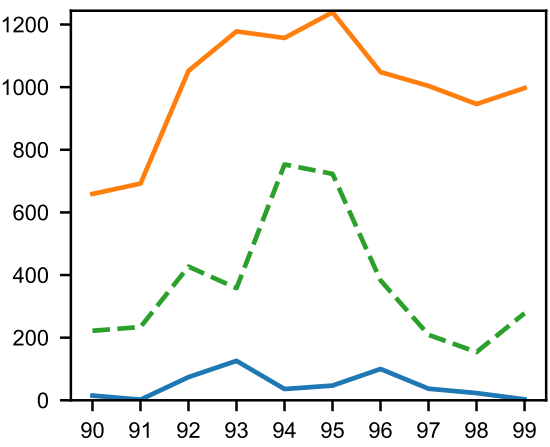

Sample allocations 2020-29 from exemplar trial Sample allocations 2020-29 from best trial
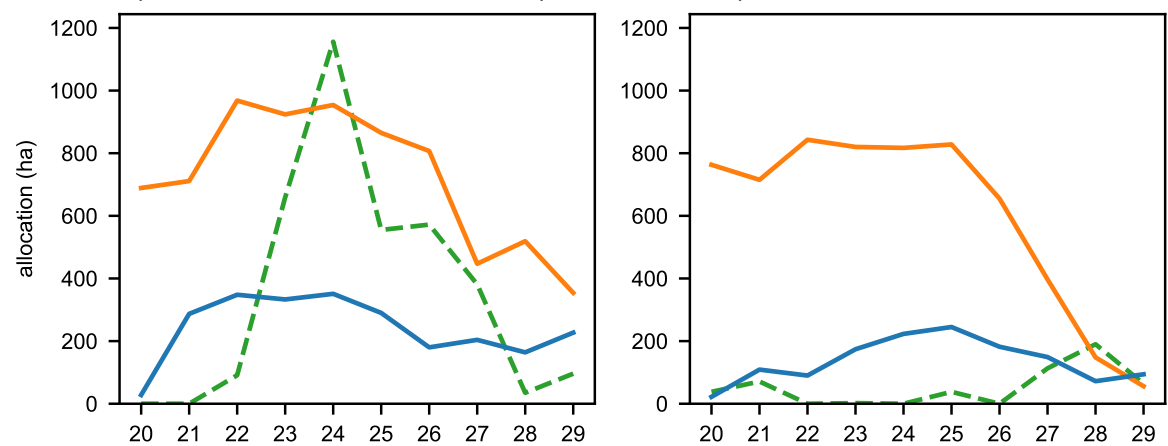

Sample allocations 2060-69 from exemplar trial

Sample allocations 2060-69 from best trial
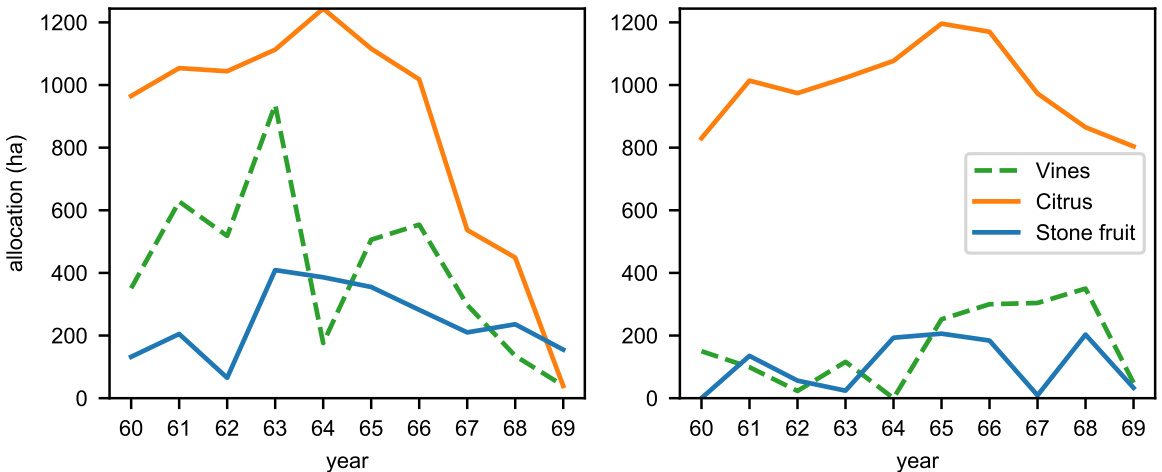

Figure 3: Annual area allocated to crops with variable productivity for maximum $N R$ solutions from the selected exemplar trials and the best performing trial, by decade 
only by $10 \%$ of their respective upper bounds.

\subsection{Discussion}

The results presented above illustrate two impacts of time on the solutions obtained to this crop allocation and water management problem: climate factors change the general setting for a planning decade, impacting on which crops, in what quantities can be planted, while the variable returns on certain crops require the solver to maintain those crops at relatively stable levels over time.

In all three decade instances, the solver is able to find at least one solution with $E F D$ equal to the minimum achievable value, given water inflows. However, across trials different solutions may be found that achieve this but with differing $N R$, which suggests that longer algorithm runs (or multiple independent runs) are required to properly explore the large search space produced by the 10-year planning horizon.

Although the total water inflow across the 1990 decade is $4 \%$ higher than that in the 2020 decade, a number of exceptionally dry months in the 1990 decade means target environmental flows were unachievable, and thus its lower bound on $E F D$ is higher than that for the 2020 decade $(276,028$ versus 183,213$)$. This allows for more profitable solutions at lower EFD in the 2020 decade. However, this decade also sees generally higher temperatures, leading to higher rates of evapotranspiration, and crop water requirements, thus only achieving the highest $N R$ with correspondingly higher EFD than in the 1990 decade.

The results obtained in our earlier work [31] relied on the classification of the year under study. Markedly different outcomes could be observed if a year was "wet", "average" or "dry", and to draw conclusions and make plans over longer time frames, some determination of the statistical distribution of these classifications over a decade was necessary. The extension described here removes this necessity, as the distribution is part of the problem parameters.

Furthermore, considering the results illustrated in Figure 2 in comparison with the limited "snapshots" presented by the analyses based on individual years [31] demonstrates the shortcomings of such an approach. For example, a wet year in the 2020 decade was able to equal the maximum $N R$ achieved in a comparable year in the 1990 decade but, over the course of the whole decade, maximum $N R$ in the 1990 decade exceeds that achievable in the 2030 decade (cf. Figure 2 points D and G.) From this we may conclude that more reliable predictions can be made on general profitability of agriculture in the MIA under changing climate conditions using the temporal modelling, and the means of achieving these outcomes. 
The temporal modelling has exposed trends in changing optimal cropping strategies over the future scenarios under climate change. It can be seen in Table 2 that crops with summer growing seasons, e.g. cotton, rice and, to a lesser extent, maize, soybean and summer vegetables, have significantly reduced cultivation in the near and far future scenarios, being replaced by winter crops. Investigation of other crops with compatible water needs and improved market access for perishable winter crops, possibly allowing their increased cultivation, may form a key part of developing climate change adaptation strategies for the region. However, there is also a general trend toward less of the land being cultivable over time, given reduced water availability and higher evapotranspiration.

It should also be noted that the current model does not yet incorporate sustainable extraction limits on river water taken for irrigation. Allowed free rein, the optimisation algorithm can, and sometimes does, reduce downstream flows to zero. This is obviously nonviable and incorporating such limits, in line with changing regulatory conditions, is an important part of future model refinement. The practical implication is that high $N R$ achieved by allowing high $E F D$ (portions of attainment surfaces adjacent to points $G$ and I in Figure 2) will not be possible. This may significantly impact decisions on future profitability and farming practices in this region.

Finally, while the solutions discovered do achieve some degree of smoothness in the year-on-year area allocated to crops with high planting and removal costs and time-varying return, it is likely that these can (and should) be refined. The results also imply that the end of the finite planning window results in decisions to remove such crops in the final planning year, an effect that will be investigated in future work over longer planning times.

\section{Conclusions}

Time permeates every facet of life, yet its treatment in optimisation problems has been limited. Temporal optimisation is a potential way to systematically incorporate time into a range of problems. In this paper, a model has been proposed that extends a crop planning and water management problem over a number of years. It allows farmers and regional planners to evaluate sustainable crop choices given projected changes in precipitation and temperature. Using specifically created decade-long data files that incorporate climate change data from the NARCLiM project, and the temporal optimisation approach, long term outcomes become more clearly discernible. This work applied a multi-objective DE to this problem and found that while climate factors determine the general setting for a 
planning decide, the variable return on certain crops require the solver to maintain their allocation of land at relatively stable levels over time. The results indicates that into the future it will no longer be sustainable to grow crops that are readily grown now, such as cotton and rice, nor to grow crops in the same quantities.

This work has the potential to be taken much further in two broad directions. Firstly, the notion of temporal optimisation needs to be extended to other problems and domains. An obvious example would be extended vehicle routing and rostering problems. In both of these, schedules could be constructed over a number of days or weeks and allow for constraints such as employees only being allowed to work for a number of days at a time. Inherent, and evident from Section 5.3, is the need for the development a set of tools that will be able to analyse multitime period results effectively. In a similar manner, it will be interesting to use other evolutionary algorithms, apart from DE, on temporal models. NSGA-II in particular, will be applied to these.

Secondly, in terms of the case study, as temporal models such as this rely on predictions into the future (in this case temperature and precipitation levels), uncertainty in the outcomes must be minimised. This essentially means that a wide variety of input data should be considered simultaneously and only solutions that have minimal deviation across these input data sets (and even in crop area size changes from one year to the next), would be considered as viable. This is in the area of robust optimisation [46, 47]. Tentative ideas and algorithms are already being pursued in this direction.

The case study model itself also requires further examination. At the present time, crops may be planted anywhere within the total area. In reality, that is not the case; certain crops require certain conditions (such as flat areas for broad acre crops like cotton while hillsides may be suitable for vines). Thus, the approach being considered is to divide the area up into a number of regions. Each region will contain characteristic information (such as soil type, elevation and slope) and will only be allowed to have one crop type planted at a time. Such an extended model will allow for realistic planning mechanisms such as crop rotation. More detailed topographic information will also allow for a realistic treatment of overland flows, notably lacking at present. Further refinement is also needed to incorporate specific factors relating to climate changes, such as alterations in crop yields and soil chemistry, which become additional temporal factors for a solver to consider. Another consideration is the development of evidence-based objectives for environmental flows, perhaps cast in terms of maximising sustainable biodiversity in downstream ecosystems, rather than arbitrary flow targets. 


\section{References}

[1] H. Beyer, B. Sendhoff, Robust optimization - A comprehensive survey, Computer methods in applied mechanics and engineering 196 (33-34) (2007) 3190-3218.

[2] E. Xevi, S. Khan, A multi-objective optimisation approach to water management, Journal of Environmental Management 77 (4) (2005) 269-277.

[3] A. Lewis, M. Randall, Solving multi-objective water management problems using evolutionary computation, Journal of environmental management 204 (2017) 179-188.

[4] N. Kurland, D. Zell, Water and business: A taxonomy and review of the research, Organization \& Environment 23 (3) (2010) 316-353.

[5] A. Singh, Simulation-optimization modeling for conjunctive water use management, Agricultural Water Management 141 (2014) 23-29.

[6] A. Singh, Conjunctive use of water resources for sustainable irrigated agriculture, Journal of Hydrology 519 (2014) 1688-1697.

[7] A. Singh, Review: Computer-based models for managing the water-resource problems of irrigated agriculture, Hydrogeology Journal (2015) 1-11.

[8] M. Falkenmark, M. Finlayson, L. Gordon, Agriculture, water, and ecosystems: Avoiding the costs of going too far, in: Water for food, water for life: A comprehensive assessment of wtar management in agriculture, Earthscan, London, UK, 2007, pp. 234-277.

[9] I. Pluchinotta, A. Pagano, R. Giordano, A. Tsoukiàs, A system dynamics model for supporting decision-makers in irrigation water management, Journal of Environmental Management 223 (2018) 815-824.

[10] E. Tijskens, H. Ramon, J. De Baerdemaeker, Discrete element modelling for process simulation in agriculture, Journal of sound and vibration 266 (3) (2003) 493-514.

[11] J. Dent, Systems simulation in agriculture, Springer Science \& Business Media, 2012. 
[12] J. Evans, F. Ji, C. Lee, P. Smith, D. Argüeso, L. Fita, Design of a regional climate modelling projection ensemble experiment-NARCliM, Geoscientific Model Development 7 (2) (2014) 621-629.

[13] J. Branke, Evolutionary optimization in dynamic environments, Vol. 3, Springer Science \& Business Media, 2012.

[14] T. Hendtlass, I. Moser, M. Randall, Dynamic problems and nature inspired meta-heuristics, in: Biologically-Inspired Optimisation Methods, Springer, 2009, pp. 79-109.

[15] S. Yang, Y. Jiang, T. Nguyen, Metaheuristics for dynamic combinatorial optimization problems, IMA Journal of Management Mathematics 24 (4) (2012) 451-480.

[16] M. Guntsch, M. Middendorf, Applying population based ACO to dynamic optimization problems, in: International Workshop on Ant Algorithms, Vol. 2463 of Lecture Notes in Computer Science, Springer, 2002, pp. 111-122.

[17] J. Branke, T. Kaußler, C. Schmidt, H. Schmeck, A multi-population approach to dynamic optimization problems, in: Evolutionary Design and Manufacture, Springer, 2000, pp. 299-307.

[18] K. Mitra, K. Deb, S. Gupta, Multiobjective dynamic optimization of an industrial nylon 6 semibatch reactor using genetic algorithm, Journal of Applied Polymer Science 69 (1) (1998) 69-87.

[19] D. Molden, Water for food water for life: A comprehensive assessment of water management in agriculture, Routledge, 2013.

[20] M. Blanco-Fonseca, G. Flichman, H. Belhouchette, Dynamic optimisation problems: Different resolution methods regarding agriculture and natural resource economics, in: Bio-Economic Models applied to Agricultural Systems, Springer, 2011, pp. 29-57.

[21] P. Reed, J. Kollat, V. Devireddy, Using interactive archives in evolutionary multiobjective optimization: A case study for long-term groundwater monitoring design, Environmental Modelling \& Software 22 (5) (2007) 683-692.

[22] G. Naadimuthu, K. Raju, E. Lee, A heuristic dynamic optimization algorithm for irrigation scheduling, Mathematical and computer modelling 30 (78) (1999) 169-183. 
[23] T. Rana, S. Khan, M. Rahimi, Spatio-temporal optimisation of agricultural drainage using groundwater models and genetic algorithms: an example from the murray irrigation area, australia, Hydrogeology journal 16 (6) (2008) 1145-1157.

[24] A. Afshar, F. Massoumi, A. Afshar, M. Mariño, State of the art review of ant colony optimization applications in water resource management, Water resources management 29 (11) (2015) 3891-3904.

[25] S. Saranya, T. Amudha, Crop planning optimization research A detailed investigation, in: IEEE International Conference on Advances in Computer Applications (ICACA), IEEE, 2016, pp. 202-208.

[26] B. Dunn, D. Gaydon, Rice growth, yield and water productivity responses to irrigation scheduling prior to the delayed application of continuous flooding in South-East Australia, Agricultural Water Management 98 (12) (2011) 1799-1807.

[27] D. Gaydon, H. Meinke, D. Rodriguez, The best farm-level irrigation strategy changes seasonally with fluctuating water availability, Agricultural Water Management 103 (2012) 33-42.

[28] K. Deb, Multi-Objective Optimization using Evolutionary Algorithms, Wiley, 2002.

[29] A. Lewis, M. Randall, S. Capon, E. Jackwitz, Constrained optimisation of agricultural water management with parameter-sensitive objectives, in: Proceedings of the $15^{\text {th }}$ International Conference on Computer Applications, 2017, pp. 79-85.

[30] J. Montgomery, A. Fitzgerald, M. Randall, A. Lewis, A computational comparison of evolutionary algorithms for water resource planning for agricultural and environmental purposes, in: Proceedings of the Congress of Evolutionary Computation, 2018, pp. 1-8.

[31] A. Lewis, M. Randall, S. Elliott, J. Montgomery, Long term implications of climate change on crop planning, in: International Conference on Computational Science, Vol. 11536 of Lecture Notes in Computer Science, Springer, 2019, pp. 369-382. 
[32] Hassall, Associates, A study of the costs of production of lucerne, medic and clover seeds in Australia, rIRDC Publication No. 01/22; RIRDC Project No. HAS-5A (2001).

URL https://rirdc.infoservices.com.au/items/01-022

[33] K. Price, R. Storn, J. Lampinen, Differential Evolution: A Practical Approach to Global Optimization, Springer, 2005.

[34] J. Montgomery, M. Randall, A. Lewis, Differential evolution for RFID antenna design: A comparison with ant colony optimisation, in: Genetic and Evolutionary Computation Conference, GECCO'11, 2011, pp. 673-680.

[35] P. Verdegaal, K. Klonsky, R. de Moura, Sample costs to establish a vineyard and produce winegrapes, Univ. California Coop. Ext., Davis.

[36] M. Hansen, Leafroll virus is costly (2014).

URL

https://www.goodfruit.com/

leafroll-virus-is-costly/

[37] K. Morgan, M. Olmstead, Peach orchard establishment and production planning budgets for Florida., in: Proceedings of the Florida State Horticultural Society, Vol. 126, Florida State Horticultural Society, 2013, pp. 35-42.

[38] I. F. T. A. (IFTA), Tasmanian stone fruit industry introduction (2005).

URL http: / / www.idfta.org/tasmanian-stone-fruit-industry/ index.html

[39] TreeFruit, Tree fruit February 2017 (2017).

URL https://www.treefruit.com.au/media/com_ acymailing/upload/treefruit_feb2017_tf.pdf

[40] B. Van den Ende, Productivity and Profitability of Growing Packham Pears, Horticulture Australia, 2003.

[41] W. Department of Primary Industries, Parks, T. Environment, Key activities report for the fortnight ending august 19 (2009).

URL https://dpipwe.tas.gov.au/Documents/RTI $\div 20002 \backslash \% 202017-18 . p d f$

[42] N. O'Connell, K. Klonsky, M. Freeman, C. Kallesen, P. Livingston, Sample costs to establish an orange orchard and produce oranges, low-volume irrigation in the San Joaquin Valley (1999). 
[43] P. Dudman, How to grow: Stone fruit (2017).

URL https://www.sbs.com.au/food/article/2012/09/

06/how-grow-stone-fruit

[44] Reserve Bank of Australia, Measures of consumer price inflation (2018).

URL https://www.rba.gov.au/inflation/measures-cpi. html

[45] D. Van Veldhuizen, Multiobjective evolutionary algorithms: Classifications, analyses, and new innovations, Technical report, School of Engineering of the Air Force Institute of Technology, Dayton, Ohio (1999).

[46] D. Bai, T. Carpenter, J. Mulvey, Making a case for robust optimization models, Management Science 43 (7) (1997) 895-907.

[47] A. Ben-Tal, L. El Ghaoui, A. Nemirovski, Robust Optimization, Princeton University Press, 2009. 